\title{
Pulse synchronization of sampled-data chaotic systems
}

\author{
Sang-Hoon Lee, ${ }^{\dagger}$ Vikram Kapila, ${ }^{\ddagger}$ and Maurizio Porfiri ${ }^{\S}$ \\ Mechanical Engineering, Polytechnic University, Brooklyn, NY 11201, USA
}

\begin{abstract}
In this paper, we consider the problem of pulse synchronization of a master-slave chaotic system in the sampled-data setting. We begin by developing a pulse-based intermittent control system for chaos synchronization. Using the discrete-time Lyapunov stability theory and the linear matrix inequality (LMI) framework, we construct a state feedback periodic pulse control law which yields global asymptotic synchronization of the sampled-data master-slave chaotic system for arbitrary initial conditions. Finally, we provide an experimental validation of our results by implementing, on a set of microcontrollers endowed with RF communication capability, a sampled-data master-slave chaotic system based on Chua's circuit.
\end{abstract}

\section{INTRODUCTION}

Secure communication systems based on synchronization of chaotic oscillators have been recently investigated, see for example [1], [2], [3], [4], [5], [6]. The general idea behind these systems is to use chaos to mask a transmitted signal, and chaos synchronization to securely recover it in reception. In this case, the chaotic oscillator at the transmitter acts as a "master" by driving the chaotic oscillator at the receiver, that consequently behaves as a "slave."

Most experimental and theoretical research on masterslave synchronization focuses on continuous-time analog systems, see for example [7], [8], [9], [10], [11], [12], [13], [14], [15], [16]. Nevertheless, in secure communication applications noise corruption in analog signal transmission can lead to severe drawbacks of synchronization schemes [17], [18]. Sampled-data systems have been studied in [3], [19], [20] to improve robustness of secure communications based on chaotic synchronization.

In a sampled-data setting, we study master-slave synchronization of chaotic oscillators that are only sporadically coupled. This scenario seems particularly realistic for communication systems. We consider the case of linear state feedback and we assume that the feedback periodically changes over time. Following the work of [9] for analog systems, we refer to this synchronization scheme as global pulse synchronization. We establish sufficient conditions for pulse synchronization using Lyapunov stability theory and the linear matrix inequality (LMI) framework. Specifically, we show that global synchronization is possible even if the oscillators are only intermittently coupled, that is, even if most of the time they are uncoupled. In order to illustrate the proposed approach, we specialize our results to the synchronization of Chua's circuits. Theoretical results are

\footnotetext{
${ }^{\dagger}$ Graduate Student

${ }^{\ddagger}$ Associate Professor

$\S$ Assistant Professor
}

validated through experiments conducted on sampled-data implementations of Chua's oscillators. Each oscillator is developed using a microcontroller and linear state feedback is realized through RF communication.

The type of intermittent coupling considered in this paper has been analyzed in the framework of consensus theory for continuous-time systems [21], [22], and peer-to-peer synchronization of analog complex networks [23], [24]. We note that, in consensus theory, the individual systems' dynamics is linear while in the present case the coupled systems are strongly nonlinear. We further observe, that results in [23], [24] are only for local synchronization since they are based on linearized dynamics. In this paper the inherent nonlinear nature of the coupled systems is retained and the problem is cast into a sampled-data setting.

The rest of the paper is organized as follows. In Section II, we present a sampled-data representation of a continuoustime chaotic system using the Euler approximation technique. In Section III, we formulate a global pulse synchronization problem. In Section IV, we provide sufficient conditions for global pulse synchronization. In Section V, we apply the LMI framework to construct periodic feedback gain matrices that yield global pulse synchronization. In Section VI, we provide experimental validation of our results using a microcontroller-based implementation of masterslave Chua's circuits. Section VII provides conclusions.

\section{Euler Approximated SAmpled-Data Chaotic SYSTEM MODEL}

In this section, we develop a sampled-data representation of a chaotic system using the Euler approximation technique. Specifically, a continuous-time chaotic system model containing a continuous nonlinear function is discretized using Euler's method to obtain a corresponding sampleddata model of the chaotic system. To begin, consider the following continuous-time chaotic system

$$
\dot{x}(t)=A_{\mathrm{c}} x(t)+g_{\mathrm{c}}(x(t)),
$$

where $x(t) \in \mathbb{R}^{n}$ is the continuous-time state vector, $A_{\mathrm{c}} \in$ $\mathbb{R}^{n \times n}$ is a constant state matrix of the continuous-time system, and $g_{\mathrm{c}}(x): \mathbb{R}^{n} \rightarrow \mathbb{R}^{n}$ is a continuous vector nonlinear function. Before proceeding, we assume that the nonlinear function, $g_{\mathrm{c}}(\cdot)$ satisfies the following condition [11]

$$
g_{\mathrm{c}}(\xi)-g_{\mathrm{c}}(\tilde{\xi})=M_{\mathrm{c}}(\xi-\tilde{\xi}),
$$

where $\xi, \tilde{\xi} \in \mathbb{R}^{n}$ and $M_{\mathrm{c}} \in \mathbb{R}^{n \times n}$ is a bounded matrix with its components dependent on $\xi$ and $\tilde{\xi}$. Next, using 
the Euler approximation method [25], the continuous-time chaotic system (1) can be discretized to yield the following sampled data representation

$$
x(k+1)=A_{\mathrm{d}} x(k)+g_{\mathrm{d}}(x(k)),
$$

where $A_{\mathrm{d}} \triangleq h A_{\mathrm{c}}+I, g_{\mathrm{d}} \triangleq h g_{\mathrm{c}}, h$ is the step size of the Euler approximation method, and $I$ is the $n \times n$ identity matrix.

Now using the unidirectional coupling technique, we characterize a chaotic slave system for (3) as follows

$$
\tilde{x}(k+1)=A_{\mathrm{d}} \tilde{x}(k)+g_{\mathrm{d}}(\tilde{x}(k))+K(k)(x(k)-\tilde{x}(k)),
$$

where $K: \mathbb{Z} \rightarrow \mathbb{R}^{n \times n}$, with $\mathbb{Z}$ denoting the set of nonnegative integers, is a feedback gain matrix that is to be designed to synchronize the slave system (4) to the master system (3).

\section{Pulse Synchronization PROBlem FOR SAMPLED-DATA CHAOTIC SYSTEM}

In order to characterize the pulse synchronization problem for the sampled-data master-slave chaotic system, we define the error between the states of the master and slave systems, (3) and (4), respectively, as follows

$$
e(k) \triangleq x(k)-\tilde{x}(k)
$$

Remark 1 Recall the definition $g_{\mathrm{d}} \triangleq h g_{\mathrm{c}}$ and that $g_{\mathrm{c}}$ satisfies (2), then it follows that $g_{\mathrm{d}}(\cdot)$ satisfies the following condition

$$
g_{\mathrm{d}}(\xi)-g_{\mathrm{d}}(\tilde{\xi})=M_{\mathrm{d}}(\xi-\tilde{\xi}),
$$

where $M_{\mathrm{d}} \triangleq h M_{\mathrm{c}}$ is $\underset{\sim}{\boldsymbol{\xi}}$ bounded matrix with its components dependent on $\xi$ and $\tilde{\xi}$.

The dynamics of the error state $e(k)$ is readily obtained from the master system dynamics (3) and the slave system dynamics (4) and is given by the following nonlinear nonautonomous system

$$
e(k+1)=\left(A_{\mathrm{d}}+M_{\mathrm{d}}-K(k)\right) e(k),
$$

where (6) has been used.

For pulse synchronization of the sampled-data masterslave chaotic system, we consider the case where $K(k)$ is a periodic gain such that $K(m N+i)=K(i)$, for $i=0,1, \ldots, p-1, K(m N+i)=K(i)=0_{n \times n}$, for $i=p, p+1, \ldots, p+q-1, N \triangleq p+q$ is the number of samples in a complete cycle, $m \in \mathbb{Z}$, and $p, q \in \mathbb{Z}_{+}$, with $\mathbb{Z}_{+}$denoting the set of positive integers. That is, over a period $N$, the periodic control gain $K(k)$ is non-zero for the first $p$ samples and zero for the next $q$ samples. In this case, the error system (7) yields

$$
e(k+1)=A(k) e(k),
$$

where

$$
\begin{aligned}
& A(k)=A_{\mathrm{d}}+M_{\mathrm{d}}-K_{k}, k=m N, \ldots, m N+p-1, \\
& A(k)=A_{\mathrm{d}}+M_{\mathrm{d}}, k=m N+p, \ldots, m N+p+q-1,(10)
\end{aligned}
$$

and $K_{k}=K(k)$ is used for notational convenience.
Finally, the problem of pulse synchronizing the dynamics of sampled-data slave system (4) to the dynamics of sampleddate master system (3) necessitates that the states of the error system dynamics given in (8) asymptotically converge to zero for any initial condition, i.e.,

$$
\lim _{k \rightarrow \infty} e(k)=0
$$

\section{SuFFicient CONDitions For Pulse SYNCHRONIZATION OF SAMPLED-DATA CHAOTIC SYSTEM}

In this section, using quadratic Lyapunov functions and Lyapunov stability analysis, we provide sufficient conditions for pulse synchronization of the sampled-data master-slave chaotic system. Before proceeding, we restrict matrix $M_{\mathrm{d}}$ to the form $M_{\mathrm{d}}=\sum_{i=1}^{\ell} \gamma_{i} \hat{M}_{i}$ where for $i=1, \ldots, \ell, \gamma_{i}$ is a bounded scalar with its components dependent on $\xi$ and $\tilde{\xi}$ and $\hat{M}_{i}$ is a constant structure matrix that captures the structure of $M_{\mathrm{d}}$. Moreover, let $\delta_{i}, i=1, \ldots, \ell$, be given scalars such that $\gamma_{i} \gamma_{j} \leq \delta_{i} \delta_{j}, i, j=1, \ldots, \ell$ for $\xi, \tilde{\xi} \in$ $\mathbb{R}^{n}$. Finally, for notational convenience, let $\mathbb{P}^{n}$ denote $n \times n$ positive definite matrices.

Theorem 1. Let $p$ matrices $K_{k}: \mathbb{Z} \rightarrow \mathbb{R}^{n \times n}, k=0, \ldots, p-$ 1 , be given and suppose there exist $N$ matrices $P_{k} \in \mathbb{P}^{n}$ such that

$$
\begin{array}{r}
2 \tilde{A}_{k}^{T} P_{k+1} \tilde{A}_{k}+2 \sum_{i=1}^{\ell} \sum_{j=1}^{\ell} \delta_{i} \delta_{j} \hat{M}_{i}^{T} P_{k+1} \hat{M}_{j}-P_{k}<0 \\
k=0, \ldots, p-1, \\
2 A_{\mathrm{d}}^{T} P_{k+1} A_{\mathrm{d}}+2 \sum_{i=1}^{\ell} \sum_{j=1}^{\ell} \delta_{i} \delta_{j} \hat{M}_{i}^{T} P_{k+1} \hat{M}_{j}-P_{k}<0 \\
k=p, \ldots, p+q-1,
\end{array}
$$

where $\tilde{A}_{k} \triangleq A_{\mathrm{d}}-K_{k}$. In this case

$$
V(e(k), k) \triangleq e^{T}(k) P_{k} e(k),
$$

is a periodic quadratic Lyapunov function, with $P_{k+N}=$ $P_{k}, k \in \mathbb{Z}$, that guarantees that the system dynamics (8) satisfy (11), thus yielding global asymptotic synchronization of the sampled-data master-slave chaotic system.

Proof. To show that the error system dynamics (8) are globally asymptotically convergent, we begin by computing the Lyapunov difference as follows

$$
\Delta V(e(k), k)=e^{T}(k+1) P_{k+1} e(k+1)-e^{T}(k) P_{k} e(k),
$$

which along the error dynamics (8) yields

$$
\Delta V(e(k), k)=e^{T}(k)\left(A^{T}(k) P_{k+1} A(k)-P_{k}\right) e(k) .
$$

Since $V(e(k), k)$ of (14) is a positive definite candidate Lyapunov function, global asymptotic stability for the error system dynamics (8) is ensured by requiring that $\Delta V(e(k), k)$ in (16) is negative definite, i.e.,

$$
A^{T}(k) P_{k+1} A(k)-P_{k}<0, k=0, \ldots, p+q-1 .
$$


Using (9) and (10) in (17) produces

$$
\begin{gathered}
\left(\tilde{A}_{k}+M_{\mathrm{d}}\right)^{T} P_{k+1}\left(\tilde{A}_{k}+M_{\mathrm{d}}\right)-P_{k}<0, k=0, \ldots, p-1, \\
\left(A_{\mathrm{d}}+M_{\mathrm{d}}\right)^{T} P_{k+1}\left(A_{\mathrm{d}}+M_{\mathrm{d}}\right)-P_{k}<0, k=p, \ldots, p+q-1 .
\end{gathered}
$$

We now provide sufficient conditions for (18) and (19) to hold. First, for $k=0, \ldots, p-1$, using $\left(\tilde{A}_{k}-\right.$ $\left.M_{\mathrm{d}}\right)^{T} P_{k+1}\left(\tilde{A}_{k}-M_{\mathrm{d}}\right) \geq 0$, we obtain

$$
\begin{gathered}
\tilde{A}_{k}^{T} P_{k+1} \tilde{A}_{k}+M_{\mathrm{d}}^{T} P_{k+1} \tilde{A}_{k}+\tilde{A}_{k}^{T} P_{k+1} M_{\mathrm{d}}+M_{\mathrm{d}}^{T} P_{k+1} M_{\mathrm{d}} \\
-P_{k} \leq 2 \tilde{A}_{k}^{T} P_{k+1} \tilde{A}_{k}+2 M_{\mathrm{d}}^{T} P_{k+1} M_{\mathrm{d}}-P_{k} .
\end{gathered}
$$

Similarly, for $k=p, \ldots, p+q-1$, using $\left(A_{\mathrm{d}}-\right.$ $\left.M_{\mathrm{d}}\right)^{T} P_{k+1}\left(A_{\mathrm{d}}-M_{\mathrm{d}}\right) \geq 0$, we obtain

$A_{\mathrm{d}}^{T} P_{k+1} A_{\mathrm{d}}+M_{\mathrm{d}}^{T} P_{k+1} A_{\mathrm{d}}+A_{\mathrm{d}}^{T} P_{k+1} M_{\mathrm{d}}+M_{\mathrm{d}}^{T} P_{k+1} M_{\mathrm{d}}$

$$
-P_{k} \leq 2 A_{\mathrm{d}}^{T} P_{k+1} A_{\mathrm{d}}+2 M_{\mathrm{d}}^{T} P_{k+1} M_{\mathrm{d}}-P_{k} .
$$

Next, using $M_{\mathrm{d}}=\sum_{i=1}^{\ell} \gamma_{i} \hat{M}_{i}$ and bound $\gamma_{i} \gamma_{j} \leq \delta_{i} \delta_{j}$, the right hand sides of (20) and (21) can be further bounded as follows

$$
\begin{array}{r}
2 \tilde{A}_{k}^{T} P_{k+1} \tilde{A}_{k}+2 M_{\mathrm{d}}^{T} P_{k+1} M_{\mathrm{d}}-P_{k} \leq 2 \tilde{A}_{k}^{T} P_{k+1} \tilde{A}_{k} \\
+2 \sum_{i=1}^{\ell} \sum_{j=1}^{\ell} \delta_{i} \delta_{j} \hat{M}_{i}^{T} P_{k+1} \hat{M}_{j}-P_{k} \\
2 A_{\mathrm{d}}^{T} P_{k+1} A_{\mathrm{d}}+2 M_{\mathrm{d}}^{T} P_{k+1} M_{\mathrm{d}}-P_{k} \leq 2 A_{\mathrm{d}}^{T} P_{k+1} A_{\mathrm{d}} \\
+2 \sum_{i=1}^{\ell} \sum_{j=1}^{\ell} \delta_{i} \delta_{j} \hat{M}_{i}^{T} P_{k+1} \hat{M}_{j}-P_{k} .
\end{array}
$$

It now follows that (12) and (13) provide sufficient conditions for (18) and (19), respectively, which in turn provide a sufficient condition for the global asymptotic stability for the error system dynamics (8).

Remark 2 Note that by selecting a periodic Lyapunov function, we are able to guarantee that the Lyapunov difference is decreasing even when the master-slave chaotic system is decoupled.

\section{LMI-BAsed Periodic Controller Synthesis For Pulse Synchronization of SAmpled-Data Chaotic SYSTEM}

In this section, we transform the sufficient condition (12) into an LMI condition which when solved along with the LMI condition (13) yields the periodic controller gain matrices $K_{k}$ of (9) to facilitate the global asymptotic pulse synchronization of the sampled-data master-slave chaotic system.

Theorem 2. Suppose there exist $N$ matrices $P_{k} \in \mathbb{P}^{n}$ and $p$ matrices $G_{k} \in \mathbb{R}^{n \times n}$ satisfying

$$
\left[\begin{array}{cc}
-\sum_{i=1}^{\ell} \sum_{j=1}^{\ell} \delta_{i} \delta_{j} \hat{M}_{i}^{T} P_{k+1} \hat{M}_{j}+\frac{1}{2} P_{k} & A_{\mathrm{d}}^{T} P_{k+1}-G_{k}^{T} \\
P_{k+1} A_{\mathrm{d}}-G_{k} & P_{k+1}
\end{array}\right]>0,
$$

and (13). In addition, for $k=0, \ldots, p-1$, let $K_{k}: \mathbb{Z} \rightarrow$ $\mathbb{R}^{n \times n}$ be given by

$$
K_{k}=P_{k+1}^{-1} G_{k}
$$

Then $P_{k}$ and $K_{k}$ satisfy (12) and (13), yielding the global asymptotic stability for the error system dynamics (8).

Proof. We begin by rewriting (12) as follows

$$
\begin{aligned}
-\sum_{i=1}^{\ell} & \sum_{j=1}^{\ell} \delta_{i} \delta_{j} \hat{M}_{i}^{T} P_{k+1} \hat{M}_{j}+\frac{1}{2} P_{k} \\
& \quad-\left(A_{\mathrm{d}}-K_{k}\right)^{T} P_{k+1} P_{k+1}^{-1} P_{k+1}\left(A_{\mathrm{d}}-K_{k}\right)>0 .
\end{aligned}
$$

Now, using $G_{k}=P_{k+1} K_{k}$ from (25), (26) becomes

$$
\begin{aligned}
-\sum_{i=1}^{\ell} & \sum_{j=1}^{\ell} \delta_{i} \delta_{j} \hat{M}_{i}^{T} P_{k+1} \hat{M}_{j}+\frac{1}{2} P_{k} \\
& -\left(A_{\mathrm{d}}^{T} P_{k+1}-G_{k}^{T}\right) P_{k+1}^{-1}\left(P_{k+1} A_{\mathrm{d}}-G_{k}\right)>0 .
\end{aligned}
$$

Finally, by an application of the Schur Complement [26], it follows that (27) is equivalent to (24). Thus, it follows that the existence of $N$ matrices $P_{k} \in \mathbb{P}^{n}$ and $p$ matrices $G_{k} \in \mathbb{R}^{n \times n}$ satisfying (24) and (13) and of $p$ matrices $K_{k}$ : $\mathbb{Z} \rightarrow \mathbb{R}^{n \times n}$ satisfying (25) is equivalent to the existence of $N$ matrices $P_{k} \in \mathbb{P}^{n}$ and $p$ matrices $K_{k}: \mathbb{Z} \rightarrow \mathbb{R}^{n \times n}$ satisfying (12) and (13). This proves that the existence of $N$ matrices $P_{k} \in \mathbb{P}^{n}$ and $p$ matrices $G_{k} \in \mathbb{R}^{n \times n}$ satisfying (24) and (13) and of $p$ matrices $K_{k}: \mathbb{Z} \rightarrow \mathbb{R}^{n \times n}$ satisfying (25) yields the global asymptotic stability for the error system dynamics (8).

Remark 3 Suppose for a given problem (24) and (13) are feasible with $p=p^{*}$ and $q=q^{*}$, respectively. Then the corresponding matrices $K_{k}, k=0, \ldots, p^{*}-1$, obtained from (25) render the error system dynamics (8) globally asymptotically stable for all $q \leq q^{*}$.

\section{ILLUSTRATIVE SYNCHRONIZATION EXAMPLE USING CHUA's System}

In this section, we illustrate the result of Section $\mathrm{V}$ by designing and implementing a periodic feedback control gain $K_{k}$ for the chaotic slave system dynamics (4). We do so by considering the sampled-data implementation of a masterslave Chua's circuit pair.

\section{A. Continuous-time Chua's System}

The continuous-time Chua's system is characterized by (1) with $n=3$,

$$
\begin{aligned}
A_{\mathrm{c}} & =\left[\begin{array}{ccc}
-\alpha & \alpha & 0 \\
1 & -1 & 1 \\
0 & -\beta & 0
\end{array}\right], \\
g_{\mathrm{c}}(x) & =\left[\begin{array}{c}
-\alpha f\left(x_{1}\right) \\
0 \\
0
\end{array}\right],
\end{aligned}
$$

where $\alpha, \beta$ are given positive scalars, $x_{1}$ denotes the first component of vector $x, f(\cdot)$ is a piecewise linear function characterized as 


$$
f\left(x_{1}\right)=b x_{1}+\frac{1}{2}(a-b)\left(\left|x_{1}+1\right|-\left|x_{1}-1\right|\right),
$$

and $a$ and $b$ are given negative constants. Referring to [11], the nonlinear function $f(\cdot)$ can be expressed as

$$
f\left(\xi_{1}\right)-f\left(\tilde{\xi}_{1}\right)=\gamma_{1}\left(\xi_{1}-\tilde{\xi}_{1}\right),
$$

where $\gamma_{1}$ is dependent on $\xi_{1}$ and $\tilde{\xi}_{1}$, and is bounded by constants $a$ and $b$ as follows $a \leq \gamma_{1} \leq b<0$.

\section{B. Euler Approximated Sampled-Data Chua's System}

Euler approximated sampled-data representations of the master and slave Chua's circuits are given by (3) and (4), respectively, with

$$
\begin{aligned}
A_{\mathrm{d}} & =\left[\begin{array}{ccc}
-h \alpha+1 & h \alpha & 0 \\
h & -h+1 & h \\
0 & -h \beta & 1
\end{array}\right], \\
g_{\mathrm{d}}(x) & =\left[\begin{array}{c}
-h \alpha f\left(x_{1}\right) \\
0 \\
0
\end{array}\right] .
\end{aligned}
$$

Note that for $g_{\mathrm{d}}$ given in (33), $M_{\mathrm{d}}$ of (6) is given by $M_{\mathrm{d}}=$ $\gamma_{1} \hat{M}_{1}$ where

$$
\hat{M}_{1}=\left[\begin{array}{ccc}
-h \alpha & 0 & 0 \\
0 & 0 & 0 \\
0 & 0 & 0
\end{array}\right] .
$$

Next, select the step size for the Euler discretization technique as $h=0.005$. Then, the sampled-data system (3) with $A_{\mathrm{d}}$ and $g_{\mathrm{d}}(x)$ given by (32) and (33), respectively, can be shown to be chaotic by showing that its largest Lyapunov exponent is positive [27]. Following the procedure of [27], which eliminates the dependence of Lyapunov exponent computation on initial condition, error magnitude, error direction, and number of iteration, the largest Lyapunov exponent is determined to be $\approx 0.4$.

\section{Numerical Parameters}

The following numerical values for the various system parameters are adopted from [11]: $\alpha=9.78, \beta=14.97, a=$ -1.31 , and $b=-0.75$. Finally, to simplify the experimental demonstration of pulse synchronization for the sampleddata master-slave chaotic system, we restrict $P_{k}$ and $G_{k}$ in (24) and (13) to be diagonal, which in turn yields diagonal gain matrices $K_{k}$ in (25). Using this procedure, for the given problem data with $p=1$, LMIs (24) and (13) were determined to be feasible up to $q=22$ and the following control gain matrix was obtained

$$
K_{1}=\operatorname{diag}(0.9511,0.9950,1.000) .
$$

Similarly, for the given problem data with $p=3$, LMIs (24) and (13) were determined to be feasible up to $q=50$ and the following control gain matrices were obtained

$$
\begin{aligned}
& K_{1}=\operatorname{diag}(0.9509,0.9948,0.9998), \\
& K_{2}=\operatorname{diag}(0.9304,1.0020,0.9779), \\
& K_{3}=\operatorname{diag}(0.9723,0.9875,1.0227) .
\end{aligned}
$$

\section{Experimental Setup}

For an experimental validation of the results of this paper, the following sequence of operations are performed.

First, the sampled-data representation of the master Chua's system (i.e., (3) with $A_{\mathrm{d}}$ and $g_{\mathrm{d}}$ given by (32) and (33), respectively) is implemented on a microcontroller (MC1). Moreover, at each sampling instant $k=0,1, \ldots$, the corresponding state $x(k)$ is transmitted by $\mathrm{MC} 1$, using an $\mathrm{RF}$ transceiver, to a second microcontroller (MC2).

Second, the sampled-data representation of the slave Chua's system (i.e., (4) with $A_{\mathrm{d}}, g_{\mathrm{d}}$, and $K$ given by (32), (33), and (35), respectively) is implemented on MC2. Note that as stated above, $\mathrm{MC} 2$ receives, using an RF transceiver, the state $x(k)$ of the master Chua's system and uses it as indicated in (4). Finally, for post-processing, at each sampling instant $k=0,1, \ldots$, MC2 communicates master and slave states $x(k)$ and $\tilde{x}(k)$, respectively, using serial communication, to Matlab running on a Personal Computer (PC). Specifically, using the serial communication capabilities of the Propeller microcontroller and Matlab (see, e.g., [28], [29] for serial interfacing of various microcontrollers and Matlab), $x(k)$ and $\tilde{x}(k)$ are imported into Matlab for a graphical representation of pulse synchronization of the sampled-data chaotic systems.

In this paper, for MC1 and MC2, we used Parallax's Propeller demo boards [30] that are based on a 32-bit processor. The Propeller demo board consists of a P8X32A-Q44 Propeller microcontroller chip, an EEPROM, and eight digital I/O pins. The P8X32A-Q44 Propeller chip has eight 32bit processors, thus allowing multi-processing. The Propeller chip can be programmed using a low-level assembly programming language or a high-level programming language Spin, used in this paper. Finally, the Propeller chip is operated with a voltage level of 3.3VDC and can communicate with a PC serially via a USB connection. For RF communication from $\mathrm{MC} 1$ to $\mathrm{MC} 2$, we used two $912 \mathrm{MHz} \mathrm{RF}$ transceivers [31], which have both transmitter and receiver functionalities on the module. The RF transceivers communicate eight bits of data at 9600 baud rate. Note that at each sampling instant $k, \mathrm{MC} 1$ executing the sampled-data representation of the master Chua's circuit needs to transmit the corresponding three-dimensional state vector $x(k)$. Since, MC1 is based on a 32-bit processor, each component of $x(k)$ is encoded using 32 bits. Thus, for the purposes of RF transmission, MC1 divides each component of $x(k)$ into four sets of eightbit data. Analogously, as MC2 receives four sets of eightbit data, it combines them to produce the original 32-bit representation of the state data transmitted by MC1.

Figure 1 shows the pair of Propeller demoboards (MC1 and MC2), with RF transceivers installed on-board, that are used to experimentally illustrate the synchronization of sampled-data master-slave Chua's system. Figure 2 shows the chaotic behavior produced by (3) running on MC1. Finally, Figures 3-a, 3-b, and 4 show that control gains (35) and (36) yield pulse synchronization of the master-slave system (i.e., (3) running on $\mathrm{MC} 1$ and (4) running on $\mathrm{MC} 2$ ) for 
$p=1, q=22$ and $p=3, q=50$, respectively. Moreover, Figures 3-c, 3-d, and 5 show that the master-slave system lacks synchronization for $p=1, q=1500$ and $p=3$, $q=2000$, with control gains (35) and (36), respectively.

\section{CONCLUSIONS}

In this paper, we developed sufficient conditions for pulse synchronization of a sampled-data master-slave chaotic system using the discrete-time Lyapunov stability theory. In addition, using the LMI framework, we constructed a periodic state feedback control law for pulse synchronization of the coupled chaotic system. We validated our results by performing pulse synchronization of a pair of Chua's circuits implemented on a pair of microcontrollers.

\section{ACKNOWLEDGMENTS}

This work is supported in part by the National Science Foundation under an RET Site grant 0227479 and a GK12 Fellows grant 0337668 and the NASA/NY Space Grant Consortium under grant 48240-7887. The authors thank Antonio Mannino for his assistance in computing Lyapunov exponents.

\section{REFERENCES}

[1] K.M. Cuomo, V.A. Oppenheim, and S.H. Strogatz, "Synchronization of Lorentz-based Chaotic Circuits with Application to Communications," IEEE Transactions on Circuits and Systems II, vol. 40, pp. 626-633, 1993.

[2] M. Feki, "An Adaptive Chaos Synchronization Scheme Applied to Secure Communication," Chaos, Solitons and Fractals, vol. 18, pp. 141-148, 2003.

[3] M. Feki, B. Robert, G. Gelle, and M. Colas, "Secure Digital Communication using Discrete-Time Chaos Synchronization," Chaos, Solitons and Fractals, vol. 18, pp. 881-890, 2003.

[4] S. Hayes, C. Grebogi, and E. Ott, "Communicating with Chaos," Physical Review Letters, vol. 70, pp. 3031-3034, 1993.

[5] B. Jovic, C.P. Unsworth, G.S. Sandhu, and S.M. Berber, "A Robust Sequence Synchronization Unit for Multi-User DS-CDMA Chaosbased Communication Systems," Signal Processing, vol. 87, pp.16921708, 2007.

[6] A.N. Miliou, I.P. Antoniades, S.G. Stavrinides, and A.N. Anagnostopulos, "Secure Communication by Chaotic Synchronization: Robustness under Noisy Conditions," Nonlinear Analysis: Real World Applications, vol. 8, pp. 1003-1012, 2007.

[7] T.L. Carroll and L.M. Pecora, "Synchronizing Chaotic Circuits," IEEE Transaction on Circuits and Systems, vol. 38, pp. 453-456, 1991.

[8] H.-H. Chen, "Global Synchronization of Chaotic Systems via Linear Balanced Feedback Control," Chaos, Solitons and Fractals, vol. 186, pp. 923-931, 2007.

[9] L. Fortuna, M. Frasca, and A. Rizzo, "Experimental Pulse Synchronization of Two Chaotic Circuits," Chaos, Solitons and Fractals, vol. 17, pp. 335-361, 2003.

[10] G. Grassi and S. Mascolo, "Nonlinear Observer Design to Synchronize Hyperchaotic Systems via a Scalar Signal," IEEE Transactions on Circuits and Systems - I: Fundamental Theory and Applications, vol. 44, pp. 1011-1014, 1997.

[11] G.P. Jiang, W.K.S. Tang, and G. Chen, "A Simple Global Synchronization Criterion for Coupled Chaotic System," Chaos, Solitons and Fractals, vol. 15, pp. 925-935, 2003

[12] R. Kilic, "Experimental Study on Impuslive Synchronization between Two Modified Chua's Circuits," Nonlinear Analysis: Real World Applications, vol. 7, pp. 1298-1303, 2006.

[13] A.I. Lerescu, N. Contandache, S. Oancea, and I. Grosu, "Collection of Master-Slave Synchronized Chaotic Systems," Chaos, Solitons and Fractals, vol. 22, pp. 599-604, 2004.

[14] J.H. Park, "Chaos Synchronization of a Chaotic System via Nonlinear Control," Chaos, Solitons and Fractals, vol. 25, pp. 579-584, 2005.
[15] L.M. Pecora and T.L. Carroll, "Synchronization in Chaotic System," Physical Review Letters, vol. 64, pp. 821-824, 1990.

[16] J. Sun and Y. Zhang, "Some Simple Global Synchronization Criterions for Coupled Time-varying Chaotic Systems," Chaos, Solitons and Fractals, vol. 19, pp. 93-98, 2004.

[17] U. Kocarev and L. Parlitz, "General Approach for Chaotic Synchronization with Applications to Communication," Physical Review Letters, vol. 74, no. 25, pp. 5028-5031, 1995.

[18] B. Andrievsky, "Adaptive Synchronization Methods for Signal Transmission on Chaotic Carrier," Mathematics and Computers in Simulation, vol. 58, pp. 285-293, 2002.

[19] T.-L. Liao and N.-S. Huang, "Dead-Beat Chaos Synchronization and its Applications to Image Communications," IEICE Transactions on Fundamentals, vol. E82-A, no. 8, pp. 1669-1673, 1999.

[20] U. Parlitz and S. Ergezinger, "Robust Communication based on Chaotic Spreading Sequences," Physics Letters A, vol. 188, pp. 146150, 1994.

[21] Y. Hatano and M. Mesbahi, "Agreement over Random Networks," IEEE Transactions on Automatic Control, vol. 50, no. 11, pp. 1867$1872,2005$.

[22] M. Porfiri and D.J. Stilwell, "Consensus Seeking over Random Weighted Directed Graphs," IEEE Transactions on Automatic Control, vol. 52, pp. 1767-1773, 2007.

[23] M. Porfiri, D.J. Stilwell, E.M. Bollt, and J.D. Skufca, "Random Talk: Random Walk and Synchronizability in a Moving Neighborhood Network," Physica D, vol. 224, pp. 102-113, 2006.

[24] D.J. Stilwell, E.M. Bollt, and D.G. Roberson, "Sufficient Condition for Fast Switching Synchronization in Time Varying Network Topologies," SIAM Journal on Applied Dynamical Systems, vol. 5, pp. 140156, 2006.

[25] M. Arcak and D. Nesic, "A Framework for Nonlinear SampledData Observer Design via Approximate Discrete-Time Models and Emulation," Automatica, vol. 40, no. 11, pp. 1931-1938, 2004

[26] S. Boyd, L. El-Ghaoui, E. Feron, and V. Balakrishnan, Linear Matrix Inequalities in System and Control Theory, Philadelphia, PA; 1994.

[27] H. Peitgen, H. Jurgens, and D. Saupe, Chaos and Fractals, SpringerVerlag, New York, NY; 1992.

[28] S.-H. Lee, Y.-F. Li, and V. Kapila, "Development of a Matlab-based Graphical User Interface for PIC Microcontroller Projects," Computers in Education Journal, vol. XV, no. 3, pp. 41-56, 2005.

[29] A. Panda, H. Wong, V. Kapila, and S.-H. Lee, "Two-Tank Liquid Level Control Using a Basic Stamp Microcontroller and a Matlab-based Data Acquisition and Control Toolbox," Computers in Education Journal, Vol. XVII, 3246, 2007.

[30] Online: http://www.parallax.com/detail.asp?product $\mathrm{id}=32100$, website of Parallax, Inc., (access link for Propeller Demo Board).

[31] Online: http://www.parallax.com/detail.asp?product $i d=27985$, website of Parallax, Inc., (access link for $912 \mathrm{MHz}$ RF Transceiver).

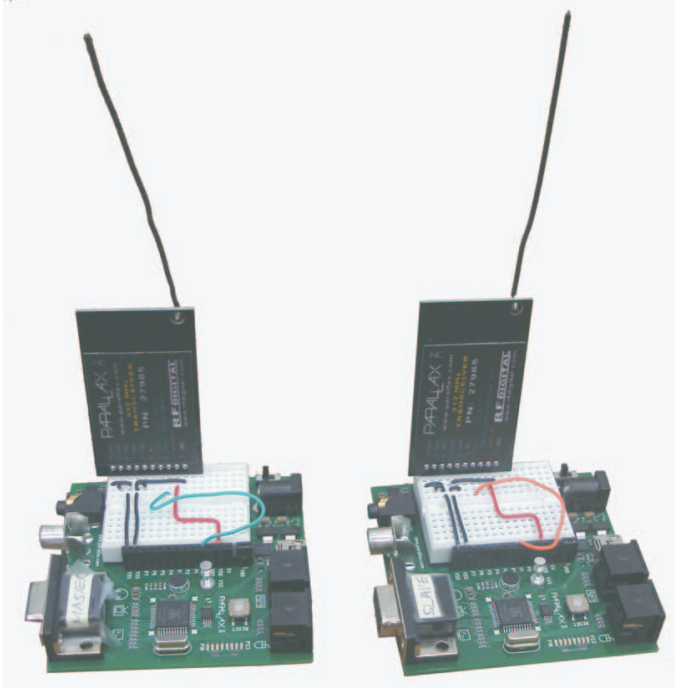

Fig. 1. Propeller demo board and $912 \mathrm{MHz}$ radio frequency transceiver for the sampled-data master-slave Chua's circuits 


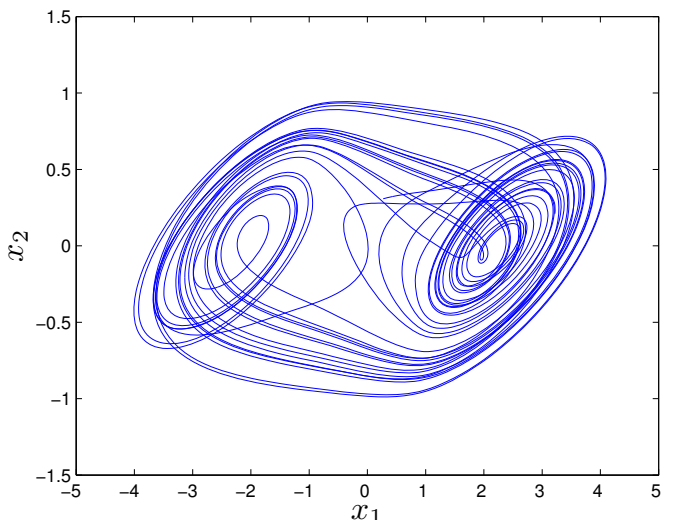

(a)

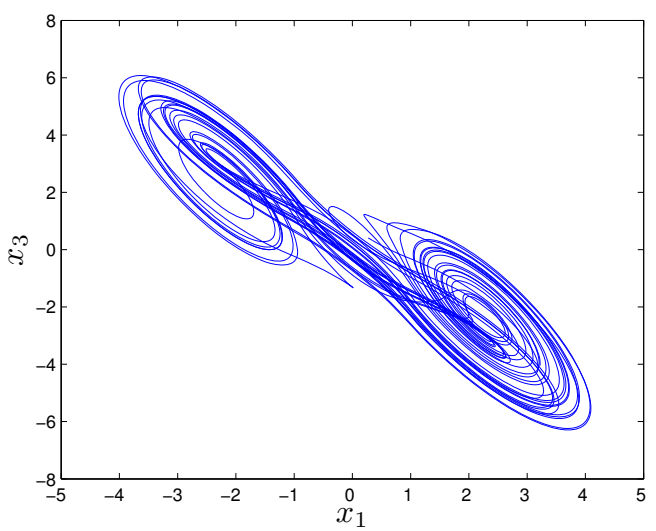

(b)

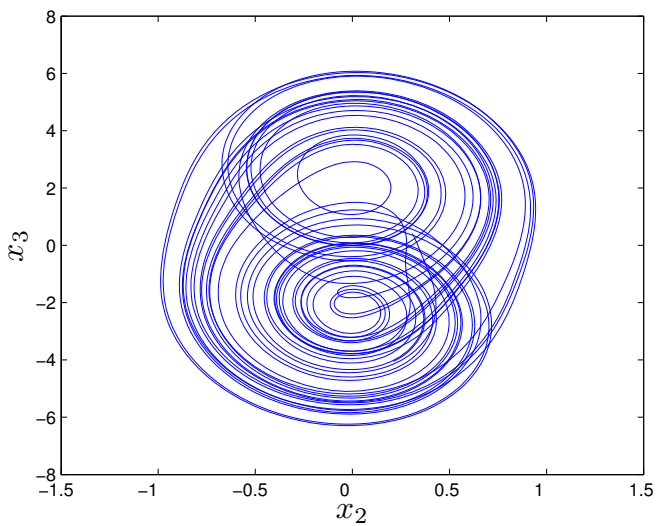

(c)

Fig. 2. Plots of the double scroll attractors of the sampled-data master Chua's circuit: (a) $x_{1} \mathrm{v} / \mathrm{s} x_{2}$, (b) $x_{1} \mathrm{v} / \mathrm{s} x_{3}$, and (c) $x_{2}$ v/s $x_{3}$
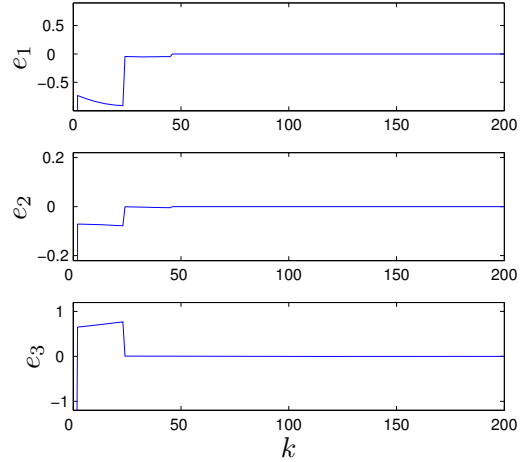

(a)
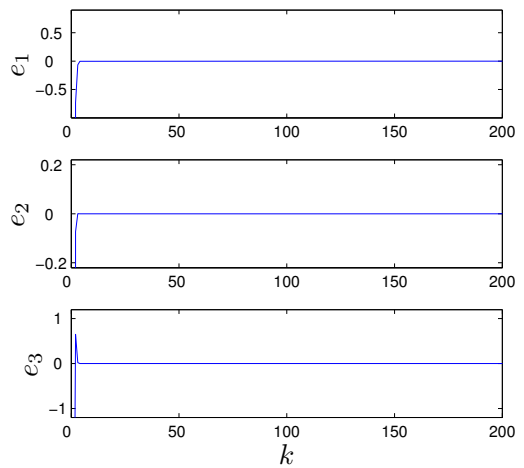

(b)
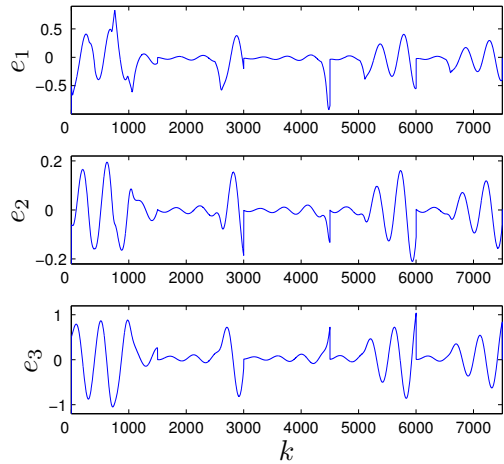

(c)
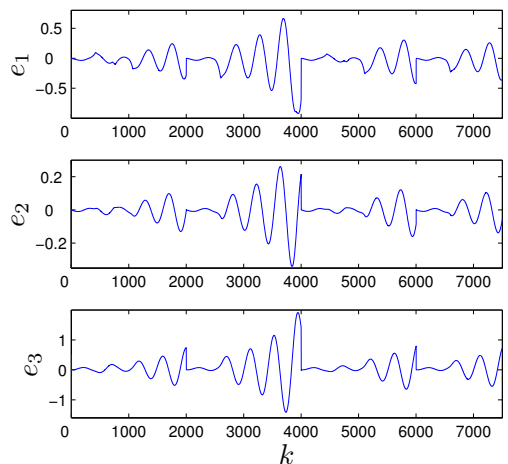

(d)

Fig. 3. Plots of the errors $\left(e_{i} \triangleq x_{i}-\tilde{x}_{i}, i=1,2,3\right)$ of the sampled-data master-slave Chua's circuits: (a) $p=1, q=22$, (b) $p=3, q=50$, (c) $p=1, q=1500$, and (d) $p=3, q=2000$ 


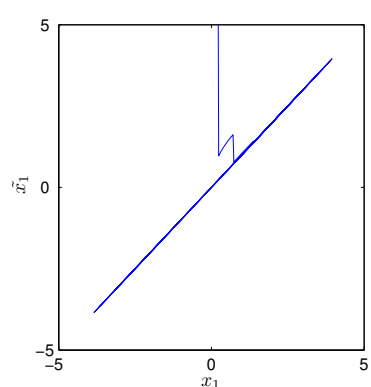

(a)

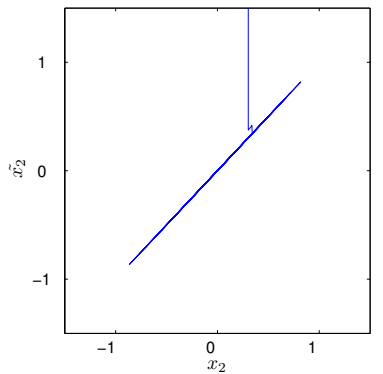

(b)

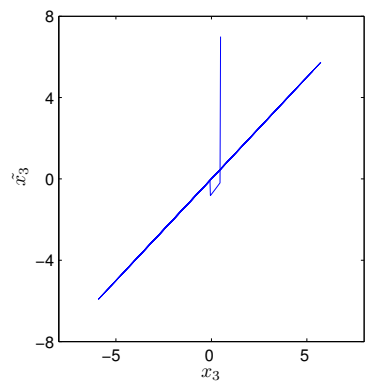

(c)

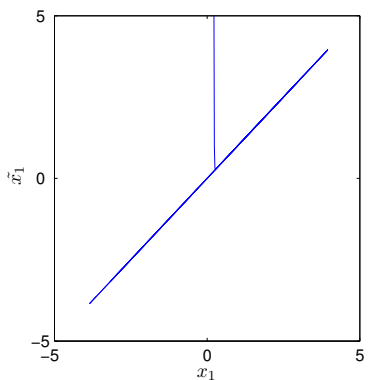

(d)

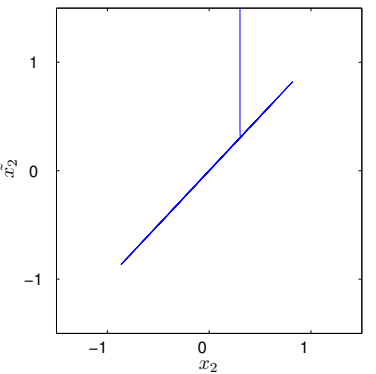

(e)

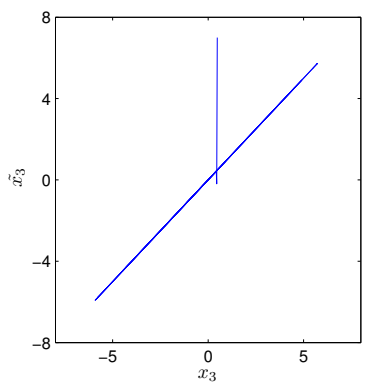

(f)

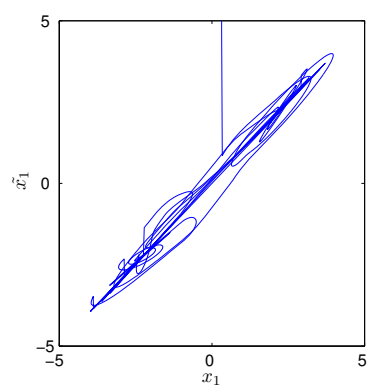

(a)

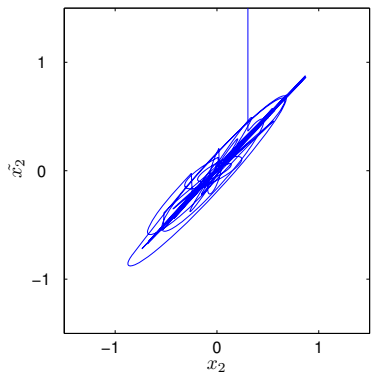

(b)

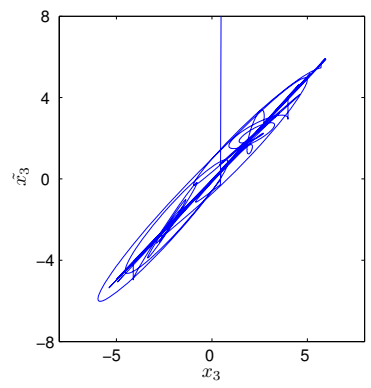

(c)

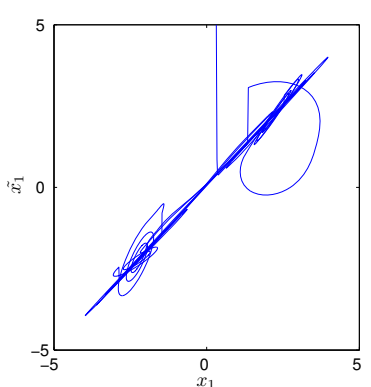

(d)

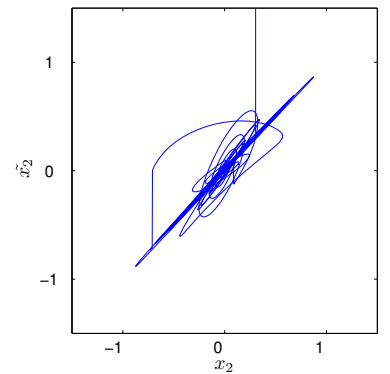

(e)

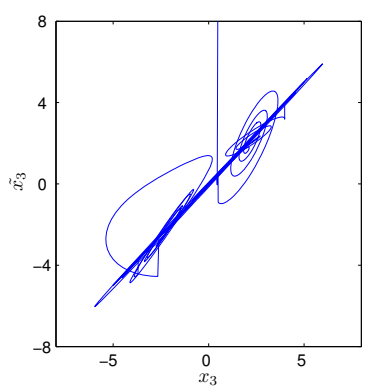

(f)

Fig. 4. Plots of the states of the sampled-data master Chua's circuit v/s the states of the sampled-data slave Chua's circuit $\left(x_{i} \mathrm{v} / \mathrm{s} \tilde{x}_{i}, i=1,2,3\right)$ showing synchronization of the master-slave Chua's circuits: (a), (b), (c) with $p=1, q=22$ and (d), (e), (f) with $p=3, q=50$

Fig. 5. Plots of the states of the sampled-data master Chua's circuit v/s the states of the sampled-data slave Chua's circuit $\left(x_{i} \mathrm{v} / \mathrm{s} \tilde{x}_{i}, i=1,2,3\right)$ showing lack of synchronization of the master-slave Chua's circuits: (a), (b), (c) with $p=1, q=1500$ and (d), (e), (f) with $p=3, q=2000$ 